UVIC-TH-07/12

UMN-TH-2618/07

FTPI-MINN-07/28

September 2007

\title{
Environmental Dependence of Masses and Coupling Constants
}

\author{
Keith A. Olive ${ }^{1}$ and Maxim Pospelov ${ }^{2,3}$ \\ ${ }^{1}$ William I. Fine Theoretical Physics Institute, \\ University of Minnesota, Minneapolis, MN 55455, USA \\ ${ }^{2}$ Department of Physics and Astronomy, University of Victoria, Victoria, BC, V8P $1 A 1$ \\ Canada \\ ${ }^{3}$ Perimeter Institute for Theoretical Physics, Waterloo, Ontario N2J 2W9, Canada
}

\begin{abstract}
We construct a class of scalar field models coupled to matter that lead to the dependence of masses and coupling constants on the ambient matter density. Such models predict a deviation of couplings measured on the Earth from values determined in low-density astrophysical environments, but do not necessarily require the evolution of coupling constants with the redshift in the recent cosmological past. Additional laboratory and astrophysical tests of $\Delta \alpha$ and $\Delta\left(m_{p} / m_{e}\right)$ as functions of the ambient matter density are warranted.
\end{abstract}




\section{Introduction}

Perhaps the most astonishing fundamental observation of the last decade was the discovery of dark energy. So far, all cosmological data are consistent with the simplest possibility: dark energy is just a new fundamental constant of nature, which does not evolve over cosmological redshifts. On the other hand, it is intriguing to think about alternative explanations associated with this profound change in infrared physics. The most straightforward way of implementing such a change is the introduction of a new ultra-light scalar degree of freedom associated with quintessence [1]. The coupling of this scalar field to matter may be the source of new cosmological phenomena such as an apparent "breakdown" of Lorentz invariance connected to the CMB frame, the existence of a "fifth force" mediated by scalar exchange, or a change of couplings and masses with time. Thus, the search for these exotic effects acquires a new actuality in the "dark energy" age.

A potential hint on the difference between the laboratory values of the fine-structure constant $\alpha[2,3]$ and the one derived from quasar absorption spectra at high redshifts [4], $\Delta \alpha / \alpha \sim-0.6 \times 10^{-5}(z \sim 0.5-3)$, has triggered a series of new phenomenological and theoretical studies of "changing couplings" [5]-[16]. Subsequent studies that employed the same "many multiplet method" have shown $\Delta \alpha$ consistent with zero with the same accuracy [17] (however, a critical discussion of this result is found in [18]). In a recent development, the comparison of $\mathrm{H}_{2}$ spectra obtained in the laboratory and at high redshifts, yielded the first hint of a possible change of $m_{p} / m_{e}$ at the three sigma level [19], whereas the analysis of the inversion spectrum of ammonium at $z=0.68$ led only to an upper limit on $\Delta\left(m_{p} / m_{e}\right)$ [20]. Null results for time evolution of couplings are reinforced by the chemical composition of Oklo rocks [21] and meteoritic abundances of rhenium, that provide stringent constraints and go back to $z \sim 0.2-0.4[22,23]$. Finally, a look into the much deeper past, to the time of the Big Bang Nucleosynthesis (BBN) [24, 25, 26], leads to the conclusion that the couplings could be different from present day values by no more than a few percent at that time.

Despite the controversial status of the non-zero claim for $\Delta \alpha / \alpha$ there have been a significant number of attempts [5]-[16], [27] - [29], to build simple models that could account for possible effects of order $O\left(10^{-5}\right)$. Theoretical models of the variation of other couplings were discussed in Refs. [30, 25]. The simplest Lagrangian that enables the variation of $\alpha$ was written down by Bekenstein [27], and involves the coupling of an ultra-light scalar field to the kinetic term in the electromagnetic Lagrangian, $\zeta_{F} \frac{\varphi}{M_{*}} F_{\mu \nu} F^{\mu \nu}$, where $M_{*}$ is a large energy scale, comparable to the Planck scale and $\zeta_{F}$ is a constant. An attractive consequence of this interaction is the absence of the evolution of the coupling in the radiation dominated epoch after the annihilation of electron-positron pairs [27]. The drift of $\alpha$ in time comes about later during the matter dominated epoch due to a nonzero, $O\left(10^{-4}-10^{-3}\right)$, 
electromagnetic contribution to the nucleon mass, which supplies a coupling of $\varphi$ to the baryon energy density. The time evolution of $\alpha$, including the sign, is calculable in terms of one free parameter, $\left(\zeta_{F} / M_{*}\right)^{2}$, and the result is a linear dependence of $\alpha$ on the logarithm of the redshift in the matter dominated epoch. Unfortunately, between redshifts $z \sim 1$ and $z=0, \Delta \alpha / \alpha$ is predicted to be far too small in the minimal model of Bekenstein; several orders of magnitude below the modern observational capabilities, once the fifth force constraints on $\zeta_{F} / M_{*}$ are taken into account. In attempt to resuscitate this model, it has been suggested that a coupling to dark matter [28, 8, 9] and/or a self-interaction potential $[10,11]$ of the scalar field can drive its evolution in a much more efficient way than the baryon energy density. Inescapably, these models have more parameters (i.e. a nearly arbitrary potential $V(\varphi)$ ), which results in a loss of predictivity. Thus almost any redshift dependence for $\alpha(z)$, including oscillatory behavior, is possible, and no concrete predictions can be obtained from these theoretical models. If the experimental limits on $\Delta \alpha$ are enforced, it has proven to be difficult to remain consistent with a substantial change in the fine structure constant at $z \sim 1$ and satisfy Oklo and meteoritic constraints. Still, fairly large classes of models (see e.g. Refs. [12], [14]- [16]) are known to pass these requirements. On the downside, if $V(\varphi)$ supplies the driving force for the scalar field, there are no convincing arguments why $\varphi$ would not evolve deep into the radiation domination epoch resulting in vastly different couplings during the BBN. In addition to these specific difficulties, there is a generic naturalness problem that all such models suffer from: it is difficult to argue that the mass of $\varphi$, as well as other terms in $V(\varphi)$, are protected to the scale of $10^{-33} \mathrm{eV}$ to allow for the requisite late evolution, and at the same time allow the $\varphi$-field to have significant couplings to matter fields. Any conceivable loop effect or nonperturbative QCD vacuum condensate would tend to induce mass parameters about twenty orders of magnitude in excess of $10^{-33} \mathrm{eV}$ (See e.g. [31]).

In all models of changing couplings discussed so far in the literature, the temporal change of $\alpha$ dominates over the possible spatial variation of $\alpha$. Although some suggestions were made that the spatial variation may dominate over temporal variations [32], no explicit models where found to date. In Bekenstein-type models, the spatial variation of couplings is caused by matter inhomogeneities and thus follows the profile of the gravitational field. If the coupling of the scalar field to matter is not stronger than the matter-gravity coupling, one expects the change in $\varphi$ to be less than the variation of the metric. For example, the difference between coupling constants on the surface of the Earth an in orbit is not going to exceed $10^{-10}$ and in practice will be much smaller once fifth force constraints are imposed. In this paper, we show that such a conclusion is not generic, and there is a whole class of models where spatial variations can be more pronounced than the cosmological variations in the recent past, opening new possibilities for searching for $\Delta \alpha$ and $\Delta m$ as functions of the matter density. 
A key to this proposal is to choose the couplings of a scalar field to matter to be much stronger than gravitational. At first glance this would appear to only worsen the problem of a fifth force induced through scalar exchange. However, this might not happen if the matter density itself leads to the effective suppression of the linear scalar field coupling to matter $[33,34]$, and/or the range of the scalar-mediated force becomes shorter than the one needed for conventional fifth-force experiments [35, 36, 37, 38]. Models that escape prohibitive fifthforce constraints may predict spatial variation of $\alpha$ and $m_{p} / m_{e}$ that exceed recent temporal variations. As we will show in the remainder of this paper, such constructions can be achieved if the dynamics of the scalar field in low-density environments is determined by its self-potential, while in regions of large overdensities, the dynamics of $\varphi$ is set by its coupling to matter. In a large subclass of such models where the matter- $\varphi$ coupling is much stronger than gravitational, the temporal evolution happens on the time scales that are much shorter than cosmological. In these models, the global temporal evolution of the scalar field could be finished a long time ago, and on average the "cosmological" values of masses and coupling constants remain constant in space and time. We denote the values of couplings and masses in rarefied low density regions as $\left\{\alpha_{r}, m_{r}\right\}$. The value of the coupling constants on Earth or in any high-density environment can be different and are labeled as $\left\{\alpha_{d}, m_{d}\right\}$. In the next sections we discuss the essential features of the model, determine observational constraints on its parameters and argue that independent tests of $\alpha_{d}-\alpha_{r}$ between different points in space are indeed warranted.

\section{Scalar field models of $\alpha(\rho)$ and $m(\rho)$}

The starting point for our analysis is the matter-gravity-scalar field action,

$$
\begin{array}{r}
S_{\phi}=\int d^{4} x \sqrt{-g}\left\{-\frac{M_{\mathrm{Pl}}^{2}}{2} R+\frac{M_{*}^{2}}{2} \partial^{\mu} \phi \partial_{\mu} \phi-V(\phi)\right. \\
\left.-\sum_{i} \frac{B_{F i}(\phi)}{4} F_{\mu \nu}^{(i)} F^{(i) \mu \nu}+\sum_{j}\left[\bar{\psi}_{j} i \not D \psi_{j}-B_{j}(\phi) m_{j} \bar{\psi}_{j} \psi_{j}\right]\right\},
\end{array}
$$

which can be viewed as a generalization of a scalar-tensor theory of gravity. In this expression, $M_{\mathrm{Pl}}=\left(8 \pi G_{N}\right)^{-1 / 2}$ is the reduced Planck mass, $\phi$ is a dimensionless scalar field with $M_{*}$ being the analogue of the Planck mass in the scalar sector. The functions $B_{F i}(\phi)$ give the $\phi$-dependence to the gauge couplings in Standard Model (SM), and the sum is extended over all SM gauge groups. $\psi_{j}$ represents Standard Model fermions that are coupled to $\phi$ via the functions $B_{j}(\phi)$. After performing a $\phi$-dependent rescaling of the matter fields, one is allowed to remove the $\phi$-dependence of the kinetic terms for the SM fermions $\psi_{i}$ and keep only couplings to the mass terms. If needed, the interaction (2.1) can be generalized further to include Higgs bosons, cold dark matter particles, etc. 
Among couplings to the SM model fields, the couplings to quarks, gluons, photons and electrons are the most important. At lower energies, we can abandon the quark-gluon description in favor of an effective coupling to nucleons and reduce (2.1) to a more tractable form,

$$
\begin{array}{r}
S_{\phi}=\int d^{4} x \sqrt{-g}\left\{-\frac{M_{\mathrm{Pl}}^{2}}{2} R+\frac{M_{*}^{2}}{2} \partial^{\mu} \phi \partial_{\mu} \phi-V(\phi)\right. \\
\left.-\frac{B_{F}(\phi)}{4} F_{\mu \nu} F^{\mu \nu}+\sum_{j=n, p, e}\left[\bar{\psi}_{j} i \not D \psi_{j}-B_{j}(\phi) m_{j} \bar{\psi}_{j} \psi_{j}\right]\right\} .
\end{array}
$$

Since we are going to consider couplings of $\phi$ that are essentially much stronger than gravitational, the stability of the model will require that $V(\phi)$ and the $B_{i}(\phi)$ functions have a minimum with respect to $\phi$. In what follows, we shall adopt the following ansatz,

$$
V(\phi)=\Lambda_{0}+\frac{1}{2} \Lambda_{2}\left(\phi-\phi_{0}\right)^{2}+\ldots ; \quad B_{i}(\phi)=1+\frac{1}{2} \xi_{i}\left(\phi-\phi_{i}\right)^{2}+\ldots
$$

where ellipses stand for cubic, quartic etc. contributions around the minima. Here $\xi_{i}, \phi_{0}$ and $\phi_{i}$ are arbitrary dimensionless numbers; $\Lambda_{0}$ and $\Lambda_{2}$ have dimensions of [Energy $]^{4}$ and we are tempted to choose $\Lambda_{0}$ to be equal to the current dark energy density to "solve" the dark energy problem.

A further simplification of the quadratic ansatz comes from the assumption that the proton and neutron $B_{p(n)}$ functions are mostly induced by the gluon $B$-function, and thus are approximately equal. With these simplifying assumptions, we can take

$$
\phi_{n} \simeq \phi_{p} \equiv \phi_{m} ; \quad \xi_{n} \simeq \xi_{p}=1 ; \quad \phi_{0}=0 .
$$

The normalization of $\xi_{p(n)}$ to one can be attained by rescaling $M_{*}$. In principle, a negative value for $\xi$ is also possible, but in this section we shall restrict our discussion to positive $\xi$ 's. Of course, the relations (2.4) are only approximate, and possible violations at the $\sim 1-10$ per mill level are naturally expected due to the nonzero quark and electromagnetic content of nucleons. The choice of $\phi_{0}=0$ can always be achieved by a constant shift of $\phi$. The ansatz (2.3) and (2.4) is very similar to the Damour-Polyakov model [34] (see also [33]), where all couplings to matter fields exhibit the same minimum. In the same vein, we assume the same minimum $\phi_{m}$ for $B_{F}(\phi)$ function. There are two important differences in our approach compared to the Damour-Polyakov models: we take $M_{*}$ to be much smaller than the Planck mass, and introduce a self-interaction potential that has a different minimum than the minimum of the $B_{i}(\phi)$ functions.

In this section we disregard higher-order nonlinear corrections to $V_{\text {eff }}$, postponing their discussion to Section 4. Furthermore, we assume a region of relatively uniform matter density $\rho$. In such regions, the scalar field equation of motion takes the following form

$$
M_{*}^{2} \square \phi+\frac{\partial V_{\text {eff }}}{\partial \phi}=0,
$$


where the effective potential is given by

$$
V_{\text {eff }}=\Lambda_{0}+\frac{1}{2} \Lambda_{2} \phi^{2}+\frac{1}{2}\left(\phi-\phi_{m}\right)^{2} \rho .
$$

This potential creates the minimum for the scalar field at

$$
\phi_{\min }=\phi_{m} \frac{\rho}{\rho+\Lambda_{2}}
$$

and the physical (canonically normalized) excitation $\varphi$ around this minimum has a mass

$$
m_{\mathrm{eff}}^{2}(\rho)=\frac{\Lambda_{2}}{M_{*}^{2}}+\frac{\rho}{M_{*}^{2}} \equiv \frac{1}{\lambda_{\mathrm{eff}}^{2}} .
$$

By definition, the longest range for the $\varphi$-mediated force is achieved in vacuum at $\rho=0$. It is instructive to present a numerical formula for $\lambda_{\text {eff }}$ at $\rho \gg \Lambda_{2}$ :

$$
\lambda_{\text {eff }}=7 \times 10^{-3} \mathrm{~cm} \times \frac{M_{*}}{1 \mathrm{TeV}}\left(\frac{10^{24} \mathrm{GeV} \mathrm{cm}^{-3}}{\rho}\right)^{1 / 2},
$$

which shows that for an extreme case with a weak-scale $M_{*}$ and terrestrial matter densities the range of the force falls under one millimeter.

If the spatial extent of the mass distribution is much larger than the Compton wavelength of the physical excitations of $\phi$ the effective interaction with a "test" nucleon takes the following form,

$$
\mathcal{L}_{\text {int }}=-m_{N} \bar{N} N\left(1+\frac{\phi_{m}^{2} \Lambda_{2}^{2}}{2\left(\Lambda_{2}+\rho\right)^{2}}-\frac{\varphi}{M_{*}} \frac{\phi_{m} \Lambda_{2}}{\left(\Lambda_{2}+\rho\right)}+\frac{\varphi^{2}}{2 M_{*}^{2}}\right),
$$

from where we can read a $\rho$-dependent mass of a nucleon,

$$
m_{N \text { eff }}=m_{N}\left(1+\frac{\phi_{m}^{2} \Lambda_{2}^{2}}{2\left(\Lambda_{2}+\rho\right)^{2}}\right)
$$

and the scalar-field-corrected Newtonian interaction potential between two nucleons separated by distance $r$,

$$
U(r)=G_{N} \frac{m_{N}^{2}}{r}\left(1+\exp \left(-m_{\mathrm{eff}} r\right) \times \frac{2 M_{\mathrm{Pl}}^{2}}{M_{*}^{2}} \frac{\phi_{m}^{2} \Lambda_{2}^{2}}{\left(\Lambda_{2}+\rho\right)^{2}}\right) .
$$

Perhaps the most interesting case to consider is $\Lambda_{2} \gg \rho$ for low density environments, such as e.g. the interstellar medium, and $\Lambda_{2} \ll \rho$ for high density environments such as stars and planets. In that case, the change in the nucleon mass and the fine structure constant can be expressed as

$$
\begin{aligned}
\frac{\Delta m_{N}}{m_{N}} & =\frac{m_{N r}-m_{N d}}{m_{N}} \simeq \frac{\phi_{m}^{2}}{2} \\
\frac{\Delta \alpha}{\alpha} & =\frac{\alpha_{r}-\alpha_{d}}{\alpha}=-\frac{\xi_{F} \phi_{m}^{2}}{2},
\end{aligned}
$$

and we assume that $\phi_{m}^{2}$ and $\xi_{F} \phi_{m}^{2}$ are much less than one. Notice that $\xi_{F}$ can be as large as $\xi_{F} \sim O(100)$ without violating the assumption that $\xi_{n} \simeq \xi_{p} \simeq 1$. 


\section{Experimental constraints on the model}

All experimental constraints on the model described by (2.10) can be divided into two broad categories. The constraints coming directly from the quadratic couplings of $\phi$ to matter to a large extent do not depend on the position of the minimum of $\phi$ and on whether this minimum can be reached for a realistic size of an overdensity in question. The second group of constraints follows from the linear coupling of $\phi$ to matter, which are very sensitive to the position of $\phi$ and on the size of the overdensity.

General remarks on chameleon-type models. Before we proceed with the analysis of our model, we would like to make several remarks regarding chameleon models. It has been shown that an appropriate choice of self-interaction potentials [35] relaxes gravitational and astrophysical constraints on the density dependent interactions of the scalar field [39] caused by a shift in the field $\phi$. Density dependent couplings could in principle cause a shift in the field value as well as its mass. However, the magnitude of the shift will depend sensitively on the local density and the length scale over which the shift occurs. For the chameleon mechanism to work, we must require that $\partial V_{\text {eff }} / \partial \phi \gg M_{*}^{2} R^{-2} \delta \phi$ or $m^{2} R^{2} \gg 1$, where $R$ is the characteristic scale of the density enhancement and $\delta \phi$ is shift in $\phi$ from the low density (cosmological) solution of $\phi$ to the local value. When this condition is satisfied, the field and its mass inside the overdensity and away from the boundary will be determined by the solution of a spacially homogeneous equation $\partial V_{\text {eff }} / \partial \phi=0$.

To begin with, let us consider models with a linear coupling to density

$$
V_{\mathrm{eff}}=V(\varphi)+\beta \rho \varphi,
$$

where we use the canonical normalization for the kinetic term of $\varphi$ and choose $\beta=M_{*}^{-1}$. In chameleon models with a quartic potential, $V(\varphi)=\lambda \varphi^{4}$, the shift in $\varphi$ is tiny as long as $\lambda$ is not tremendously small. The effective mass when $\partial V_{\text {eff }} / \partial \varphi=0$ is $\lambda^{1 / 6}(\beta \rho)^{1 / 3}$ and for $\lambda \sim O(1)$ is far greater than $R_{\oplus}^{-2}$. In this case, as for all stiff potentials, the local environment determines the field dependent couplings. Another example is the quintessencelike potential $V(\varphi)=M^{5} / \varphi$ with $M \sim 10^{-24} M_{\mathrm{Pl}}$. The cosmological background solution for $\beta \sim M_{\mathrm{Pl}}^{-1}$ gives $\varphi \sim M_{\mathrm{Pl}}$ so that the potential and cosmological density are of the same order of magnitude. Naively, the local solution of $\partial V_{\text {eff }} / \partial \varphi=0$ would yield $\varphi^{2}=M^{5} M_{\mathrm{Pl}} / \rho_{\oplus} \sim 10^{-27} M_{\mathrm{Pl}}^{2}$ taking $\rho_{\oplus} \sim 10^{-93} M_{\mathrm{Pl}}^{4}$. However in this case, the gradient term in eq. (2.5) dominates and the correct solution for the local value of $\phi$ is a small shift from the background value of order $\delta \varphi \sim \rho_{\oplus} R_{\oplus}^{2} / M_{\mathrm{Pl}}$ or $\delta \varphi \sim g R_{\oplus} M_{\mathrm{Pl}} \sim 10^{-9} M_{\mathrm{Pl}}$ where $g$ is the local acceleration on the Earth's surface. In this case, the chameleon mechanism is not operative. It can be restored if one adds a constant, $M^{4}$ to $V(\varphi)$. By doing so, the mass scale, $M$, can be made significantly smaller $M \sim 10^{-30} M_{\mathrm{Pl}}$ [36] and now the background solution for $\varphi$ yields a very small value $\varphi \sim 10^{-15} M_{\mathrm{Pl}}$. In this case, the gradient terms can be safely neglected and the local solution for $\varphi$ is indeed given $\partial V_{\text {eff }} / \partial \varphi=0$. A 
similar argument can be made to show that the local density has virtually no effect on the background field value for potentials of the form $V(\varphi)=-\mu^{4} \ln \left(\varphi / M_{\mathrm{Pl}}\right)$ with $\mu \sim 10^{-3} \mathrm{eV}$ that was discussed recently in [40].

Exact solutions for spherical (under)overdensities. The model based on the effective potential (2.6) considered in this paper is simple to analyze, as the field equations are linear. This allows us find an analytic solution for a spherical region of constant density, and consider both large and small $M_{*}$ limits. The general form of the solution for $\phi$ as the function of radius for a spherical region of density $\rho_{1}$ of maximal extent $R$ surrounded by the infinite region of density $\rho_{2}$ takes the following form:

$$
\phi(r)=\left\{\begin{array}{cc}
\phi_{1}+\frac{A}{r} \sinh \left(m_{1} r\right), & r<R \\
\phi_{2}+\frac{B}{r} \exp \left(-m_{2} r\right), & r>R
\end{array}\right.
$$

where the constants of integration

$$
\begin{array}{r}
A=\frac{\left(\phi_{2}-\phi_{1}\right)\left(1+R m_{2}\right)}{m_{2} \sinh \left(m_{1} R\right)+m_{1} \cosh \left(m_{1} R\right)} ; \\
B=\exp \left(m_{2} R\right) \times \frac{\left(\phi_{2}-\phi_{1}\right)\left(\sinh \left(m_{1} R\right)-R m_{1} \cosh \left(m_{1} R\right)\right)}{m_{2} \sinh \left(m_{1} R\right)+m_{1} \cosh \left(m_{1} R\right)} ;
\end{array}
$$

are uniquely determined by boundary conditions, for which the physical choice is $\phi(\infty)=\phi_{2}$ and $\phi^{\prime}(0)=0$. In these formulae, $m_{1}, m_{2}$, and $\phi_{1}, \phi_{2}$ are the mass and vacuum expectation values calculated according to (2.8) and (2.7) for $\rho=\rho_{1}, \rho_{2}$. Eq. (3.2) is a generalization of the solution previously found in Ref. [39].

Adapting this solution to the case of $\rho_{1}=\rho_{\oplus}$ and $R=R_{\oplus}$, it is easy to see that in the limit of large $M_{*}$, or more precisely $m_{1} R_{\oplus} \simeq \rho_{\oplus}^{1 / 2} M_{*}^{-1} R_{\oplus} \ll 1$, the solution simplifies to a quadratically rising function on the inside and a $1 / r$-falling function on the outside (for simplicity, we also take $\Lambda_{2}, \rho_{2} \rightarrow 0$ leading to $\phi_{2}=0$ ),

$$
\phi(r)=\left\{\begin{array}{c}
-\phi_{1}\left(\frac{m_{1}^{2} r^{2}}{6}-\frac{m_{1}^{2} R^{2}}{2}\right), \quad r<R ; \\
\phi_{1} \frac{m_{1}^{2} R^{3}}{3 r}, \quad r>R .
\end{array}\right.
$$

It is easy to see that this solution exactly follows the gravitational potential profile, with $\phi_{1}=\phi_{m}$. The change in the nucleon mass between $r=R_{\oplus}$ and spatial infinity is

$$
\frac{\Delta m_{N}}{m_{N}}=\frac{1}{2}\left(\phi^{2}(\infty)-\phi^{2}\left(R_{\oplus}\right)\right) \sim\left(g R_{\oplus}\right) \times \frac{M_{\mathrm{Pl}}^{2} \phi_{m}^{2}}{M_{*}^{2}} \sim\left(10^{-9}-10^{-8}\right) \times \frac{\phi_{m}^{2} M_{\mathrm{Pl}}^{2}}{M_{*}^{2}} .
$$

which is exactly what one anticipates in a linearized scalar-tensor theory of gravity with the relative strength of spin-0 to spin- 2 exchange given by $2 M_{\mathrm{Pl}}^{2} \phi_{m}^{2} / M_{*}^{2}$. Furthermore, this 
limit, namely $\rho_{\oplus}^{1 / 2} M_{*}^{-1} R_{\oplus} \ll 1$ is guaranteed in our model as long as $M_{*}>10^{13} \mathrm{GeV}$. This regime is of no further interest to us in this paper, as it has been investigated in a number of previous publications.

The opposite regime is achieved when $m_{1} R \gg 1$, in which case the interior solution quickly adjusts to $\phi=\phi_{1}$ for $r<R$. Moreover, if in addition $m_{2} R \gg 1$, the spherical symmetry of the problem becomes irrelevant, and the solution degenerates into $\phi$ being frozen to its respective minima, $\phi_{1}$ and $\phi_{2}$, everywhere in space except for a small region near the surface separating two density regions. Defining this surface as $z=0$, we can write down a simplified form of (3.2) which now takes the form:

$$
\phi(x)=\left\{\begin{array}{cc}
\phi_{1}+\frac{\left(\phi_{2}-\phi_{1}\right) m_{2}}{m_{1}+m_{2}} \exp \left(m_{1} z\right), & z<0 \\
\phi_{2}+\frac{\left(\phi_{1}-\phi_{2}\right) m_{1}}{m_{1}+m_{2}} \exp \left(-m_{2} z\right), & z>0 .
\end{array}\right.
$$

A smooth boundary would lead to an adiabatic adjustment of $\phi$ between its minima provided that the Compton length of the scalar is much less than the characteristic scale of density change. For the atmosphere this scale is given by $\Delta_{a t m}=1 /\left|d \log \left(\rho_{a t m} / \rho_{\oplus}\right) / d z\right| \sim 1 \mathrm{~km}$, and the adjustment of the scalar field will occur as long as

$$
\lambda_{\text {eff }}\left(\phi_{a t m}\right) \ll \Delta_{a t m}
$$

ensuring that in a terrestrial laboratory environment $\phi$ is exponentially close to $\phi_{\min }(2.7)$. Neglecting the small $\Lambda_{2}$-proportional contribution to the scalar field mass, we find that the condition (3.7) implies that

$$
M_{*} \ll 1 \mathrm{~km} \times \rho_{a t m}^{1 / 2} \Longrightarrow M_{*} \ll 10^{9} \mathrm{GeV}
$$

Clearly, this choice of $M_{*}$ selects scalar models that are significantly more strongly coupled than $M_{\mathrm{Pl}}$-normalized models and therefore the behavior of $\phi$ on Earth will depart drastically from the gravitational potential $\sim g_{00}-1$. Next we explore whether the choice of strong coupling (3.8) can survive gravitational and astrophysical constraints.

Astrophysical constraints. First we discuss the astrophysical constraints on the model which employs a $\phi^{2}$ coupling to photons and nucleons (the linear coupling is suppressed by $\left(\Lambda_{2} / \rho_{1}\right)^{2}$ and is assumed to be $\left.\ll 1\right)$. It is clear that the quadratic coupling will be less severely constrained than a linear coupling by the thermal emission rate of $\phi$-quanta from the hot interiors of stars. Indeed, the overall emission rate scales as $M_{*}^{-4}$ rather than $f_{a}^{-2}$ as one would routinely find in an axion-type model. As a result, instead of a lower limit to $f_{a}$ or order $10^{9}-10^{10} \mathrm{GeV}$, we expect to find a much more relaxed bound on $M_{*}$, of the order of the electroweak scale.

Let us calculate the emissivity of $\phi$ quanta due to pair annihilation of photons. The amplitude for this process is induced by the $\xi_{F} \phi^{2} F_{\mu \nu} F^{\mu \nu}$ term in the Lagrangian, leading 
to a cross section for this process in the center of mass frame,

$$
\sigma_{\gamma \gamma \rightarrow \phi \phi}=\frac{\xi_{F}^{2}}{M_{*}^{4}} \frac{\omega^{2}}{32 \pi} .
$$

This cross section results in an energy loss (Energy/volume/time) for a thermalized gas of photons at the level of

$$
\Gamma_{\gamma \gamma \rightarrow \phi \phi}=n_{\gamma}^{2}\left\langle 2 \omega \sigma_{\gamma \gamma \rightarrow \phi \phi}\right\rangle=\frac{\zeta(3) \pi}{63} \frac{\xi_{F}^{2} T^{9}}{M_{*}^{4}} \simeq 0.06 \times \frac{\xi_{F}^{2} T^{9}}{M_{*}^{4}} .
$$

Comparing this to the typical limit on $\Gamma=\epsilon_{x} \rho_{\text {core }}<10^{-14} \mathrm{MeV}^{5}$ that follows from the constraints on the emissivity of light particles in cores of supernovae [41],

$$
\epsilon_{x} \lesssim 10^{19} \mathrm{erg} \mathrm{g}^{-1} \mathrm{~s}^{-1} \text { at } \rho_{\text {core }}=3 \times 10^{14} \mathrm{~g} \mathrm{~cm}^{-3}, \quad T_{\text {core }}=30 \mathrm{MeV} \text {, }
$$

we obtain a typical sensitivity to the coupling of $\phi$ to photons,

$$
M_{*} \xi_{F}^{-1 / 2} \gtrsim 3 \mathrm{TeV} .
$$

This limit is admittedly not very precise, as it is quite sensitive to the temperature of the core, and more conservative assumptions about $T_{\text {core }}$ may result in relaxation of (3.12) by a factor of a few. Other channels of $\phi$-production from light species, such as $\gamma e \rightarrow e \phi \phi$ or $e^{+} e^{-} \rightarrow \phi \phi$ will be further suppressed by the smallness of electromagnetic couplings or by the ratio of $m_{e} / T$.

The constraint (3.12) is not far the limits on the 6-dimensional Planck scale $M_{6}$ in models with two large extra dimensions where gravity is allowed to propagate $[42,43,44]$ in extra dimensions. This is not a total coincidence: in models with two large extra dimensions the total emissivity of Kaluza-Klein gravitons also scales as $M_{6}^{-4}$. We note that due to the high power of temperature in the emission rate (3.10) the supernovae constraints are expected to be superior to other astrophysical constraints from energy loss mechanisms derived from the considerations of red giants, old neutron stars, etc. (See Ref. [41] for further details).

Similar considerations can be applied to the bremsstrahlung-like emission process $N+$ $N \rightarrow N+N+\phi+\phi$, where again pairs of $\phi$ are emitted. This process is important because the density of neutrons inside the core is rather large. Here, instead of performing a detailed calculation which is perhaps not warranted, we use a simple Weizsacker-Williamstype estimate for the energy loss. Specifically, we estimate the probability of energy loss in a collision of two nucleons to be the product of elastic nucleon-nucleon cross section $\sigma_{N N}$ and the probability of an emission of certain amount of energy into $\phi$-quanta by an initial or final state nucleon,

$$
\langle E \sigma v\rangle \sim \frac{1}{12 \pi^{4}} \frac{T^{3} m_{N}^{2}}{M_{*}^{4}}\left(\frac{T}{m_{N}}\right)^{1 / 2} \sigma_{N N}
$$


which leads to an energy loss of

$$
\Gamma_{N N \rightarrow N N \phi \phi} \sim \sigma_{N N} \times \frac{n_{N}^{2} T^{7 / 2} m_{N}^{3 / 2}}{12 \pi^{4} M_{*}^{4}},
$$

where and $n_{N}$ is the number density of neutrons $\simeq \rho / m_{N}$. For the relevant range of energies $\sigma_{N N}$ can be taken on the order of $25 \mathrm{mbn}$ [44]. Using the same parameters as before (3.11), we arrive at the constraint on $M_{*}$,

$$
M_{*} \gtrsim 15 \mathrm{TeV}
$$

which is very similar to (3.12). With these constraints, we conclude that the effective range of $\varphi$-force in terrestrial environment (2.9) can indeed be as short as a millimeter.

Gravitational force constraints. Gravitational force constraints on the model are by far the most complicated as they depend very sensitively on the effective distance range this force is probed at.

Could the laboratory measurements of the gravitational force improve over the astrophysical bounds (3.12)? First, we look at the exchange by two quanta of $\varphi$ that does not depend on $\phi_{\min }$. Such an exchange leads to a $1 / r^{3}$ potential,

$$
V=-\frac{1}{r^{3}} \frac{m_{N}^{2}}{64 \pi^{3} M_{*}^{4}}
$$

which is limited by recent searches for deviations from the gravitational $1 / r$ behavior at short distances [45]. Specifying the constraints on phenomenological coefficient $\beta_{3}$ from Ref. [46] to our model prediction (3.16), we arrive at

$$
V=-\beta_{3} \frac{G_{N} m_{N}^{2}}{r} \frac{1 \mathrm{~mm}^{2}}{r^{2}} \text { with } \beta_{3}<1.3 \times 10^{-4} \Longrightarrow M_{*}>2 \mathrm{TeV}
$$

which is very close to the astrophysical bounds (3.12) and (3.15). We note again that the transition from a $1 / r$ to a $1 / r^{3}$ potential that may occur in our model at short distances is very similar to the transition expected in theories with two large extra dimensions.

Constraints from the Yukawa part of (2.12) are somewhat less straightforward to implement. For a range of $O\left(10^{-2}-1 \mathrm{~cm}\right)$ in a medium, the constraint on its strength [46] specialized to our case with the use of (2.12) takes the following form

$$
\frac{\phi_{m}^{2} m_{N}^{2} \Lambda_{2}^{2}}{4 \pi M_{*}^{2} \rho^{2}} \lesssim \text { few } \times 10^{-40},
$$

or

$$
\frac{\phi_{m}^{2}}{10^{-6}} \times\left(\frac{\mathrm{TeV}}{M_{*}}\right)^{2} \times\left(\frac{\Lambda_{2}}{\mathrm{eV}^{4}}\right)^{2} \lesssim 10^{12}-10^{13}
$$


where we took $\rho \simeq 10 \mathrm{~g} / \mathrm{cm}^{3}$ for the density of molybdenum used in experiments of Ref. [45]. Perhaps an even more convenient from of the same constraint arises when we trade $\Lambda_{2}^{1 / 2} / M_{*}$ for $1 / \lambda_{\text {vac }}$, the range of $\phi$-force in the vacuum,

$$
\frac{\phi_{m}^{2}}{10^{-6}} \times\left(\frac{M_{*}}{\mathrm{TeV}}\right)^{2} \times\left(\frac{\mathrm{km}}{\lambda_{\text {vac }}}\right)^{4} \lesssim 10^{3}-10^{4} .
$$

Constraints (3.19) and (3.20) do not look intimidatingly stringent, and indeed can be satisfied by an appropriate choice of $M_{*}, \phi_{m}$ and $\Lambda_{2}$. It is also important to note that these constraints can be satisfied with a relatively short-range $\phi$-mediated force in vacuum, that could be shorter in range than e.g. typical distances within the solar system.

Clock comparison constraints and constraints on the variations of couplings are of particular interest to us in this paper. The now classic comparison of atomic clocks at an altitude of $R_{\text {orbit }}=10^{4} \mathrm{~km}$ with clocks on the ground have produced the limit of $2 \times 10^{-4}$ on possible deviations from predictions of general relativity [47] (as quoted in the review $[48])$,

$$
\frac{\left|\Delta \omega_{\mathrm{H}}\right|}{\omega_{\mathrm{H}}} \lesssim 2 \times 10^{-4} \times\left|\Phi\left(R_{\oplus}\right)-\Phi\left(R_{\text {orbit }}\right)\right| \sim 5 \times 10^{-13},
$$

where $\Delta \omega_{\mathrm{H}}$ is the extra frequency shift of the Hydrogen maser added to the shift predicted by general relativity, and $\Phi(r)$ is the gravitational potential at distance $r$ from Earth's center. In our model, the difference between clock frequency on the ground and in orbit would receive an additional correction from the difference of coupling constants and masses caused by $\Delta \phi$ :

$$
\frac{\Delta \omega_{\mathrm{H}}}{\omega_{\mathrm{H}}}=\frac{\Delta\left(\alpha^{4} m_{e}^{2} g_{p} m_{p}^{-1}\right)}{\alpha^{4} m_{e}^{2} g_{p} m_{p}^{-1}}=-\left(\frac{\Delta \phi^{2}}{2}\right) \times\left(1-2 \xi_{e}+4 \xi_{F}\right),
$$

where for simplicity we assumed the same scaling for $\Lambda_{Q C D}$ and quark masses with $\phi$ which keeps the proton $g$-factor $g_{p}$ fixed as a function of distance. The density of a medium surrounding the satellite is certainly very low, and thus it is tempting to take $\Delta \phi^{2}=\phi_{m}^{2}$. Note however, that if the in-medium range of the force is much shorter than a typical scale of a satellite, $L_{\text {sat }} \sim 1-10 \mathrm{~m}$, with its average density being $M_{\text {sat }} / L_{\text {sat }}^{3} \sim 100 \mathrm{~kg} / \mathrm{m}^{3} \sim$ $0.1 \mathrm{~g} / \mathrm{cm}^{3}$, the scalar field inside a satellite will roll back to its in-medium value, resulting in an exponential suppression of $\Delta \phi$. Therefore, we expect the clock comparison constraint to be at the level of

$$
\phi_{m}^{2} \exp \left(-2 L_{\text {sat }} / \lambda_{\mathrm{eff}}\right) \times\left|1-2 \xi_{e}+4 \xi_{F}\right| \lesssim 10^{-12},
$$

noting that the precise amount of exponential suppression would also depend on the position of clocks inside the satellite. Although very powerful for $M_{*} \gtrsim 10^{8} \mathrm{GeV}$ when the exponential factor is of order one, eq. (3.23) is not particularly constraining for an interesting range of $1 \mathrm{TeV}<M_{*}<100 \mathrm{TeV}$, as the value of $\phi$-field inside the satellite would be very close to that on the surface of the Earth. 
Straightforward constraints on $\phi_{m}^{2}$ can be deduced from the comparison of coupling constants measured in cosmological settings and in the laboratory. Interpreting the null results of [17], $|\Delta \alpha / \alpha|<10^{-5}$, in terms of parameters of our model, we obtain the constraint

$$
\left|\xi_{F}\right| \phi_{m}^{2}<2 \times 10^{-5}
$$

However, should one accept the criticism expressed in Ref. [18], and interpret the result in [4] as a non-zero value of $\Delta \alpha$, then one predicts $\xi_{F} \phi_{m}^{2} \sim 10^{-5}$, and the sign of $\xi_{F}$ comes out to be positive (for positive $\xi_{F}$, the low density environments have a larger coefficient in front of $F_{\mu \nu}^{2}$ and therefore a smaller value of $\alpha$ in agreement with [4]). In a similar fashion, the indication of a non-zero $\Delta\left(m_{e} / m_{p}\right)$ [19] can be interpreted as a nonzero value for the $\left(\xi_{e}-\xi_{p}\right) \phi_{m}^{2}$ combination. Another interesting possibility occurs for a choice of parameters when $\rho_{\oplus} \gg \Lambda_{2} \gtrsim \rho_{q s o}$, where $\rho_{q s o}$ is the average density in a quasar absorption system. In this case, not only does one expect a variation in the couplings measured in these systems, but one would expect a shift in $\alpha$ from one absorption system to another as function of their density $^{1}$. An additional analysis of data in Refs. [4] and [17] searching for $\alpha(\rho)$ correlation might be warranted.

It is also very important to stress that the Oklo constraint on $\Delta \alpha$ does not carry any weight in our model. Indeed, the Oklo phenomenon obviously occurred in large density environment, which means that $\phi=\phi_{m}$ with good accuracy back at the time the Oklo reactor was active, as well as it is now. As to the constraints from meteorites [22], similarly to eq. (3.23), one expects an exponential suppression of the effect by the density of meteorite, as long its size is larger than the $\phi$-field penetration length, $L_{m e t}>\lambda_{\text {eff }}$.

Cosmological constraints. Cosmology can constrain the presence of new degrees of freedom in the Universe. Big Bang Nucleosynthesis can in principle impose a constraint on a number of new relativistic degrees of freedom that carries a comparable amount of entropy as photons or neutrinos. It is very easy to see, however, that even if $\phi$ is initially thermally excited, its decoupling occurs well before the neutrino decoupling because $M_{*}^{-2} \ll G_{F}$. Since traditionally the BBN constraints are expressed in terms of the number of "new neutrino species", we can immediately conclude that $\phi$ contributes to this number as $4 / 7$ or less and thus cannot be ruled out on the grounds of light element abundances [26]. It is interesting to note that the position of $\phi$ during the time of $\mathrm{BBN}$ is close to $\phi_{m}$, as the energy density of nonrelativistic matter during $\mathrm{BBN}$ is comparable to the terrestrial $\rho$ making the BBN sensitivity to $\Delta \alpha$ far less than (3.24).

The late time evolution of $\phi$ can be rather uninteresting. The scalar field remains at $\phi=\phi_{m}$ until the moment when $\rho$ drops below $\Lambda_{2}$, after which it settles towards $\phi=0$.

\footnotetext{
${ }^{1}$ In this sense, the observed scatter in $\alpha$ may be real and due to a local environmental effect. In this case, a variation of the density within the absorber. A related solution (which does not require new physics) accounted for the scatter through another environmental effect, namely the variation of the isotopic abundance of $\mathrm{Mg}$ [49].
} 
Since $M_{*} \ll M_{\mathrm{Pl}}$, the vacuum mass $m_{0}=\Lambda_{2}^{1 / 2} / M_{*}$ is much larger than the Hubble scale at which $\rho \sim \Lambda_{2}$ and any oscillations around the minimum are very efficiently damped. Thus, the transition from $\phi=\phi_{m}$ to to $\phi=0$ was completed when $\rho \sim \Lambda_{2} \sim \mathrm{eV}^{4}$ at redshifts $z \sim 10^{3}-10^{4}$. The amount of energy released in such a transition is $\sim \phi_{m}^{2} \Lambda_{2}$, and has a negligible effect on the expansion history, as $\phi_{m}^{2}$ is constrained to be much less than 1 .

An interesting possibility emerges when $\Lambda_{2}$ is comparable to the matter energy density at redshifts $z \sim 1$. Then, the evolution of the coupling constants in time towards the minimum at $\phi=0$ would occur on cosmological scales, while remaining frozen at $\phi \simeq \phi_{m}$ on galactic scales where the matter energy density is larger than the average cosmological energy density by many orders of magnitude. This choice of parameters serves as a concrete realization of scenario proposed in [50], where it was suggested that the cosmological evolution of $\alpha$ may proceed in some sense independently from the evolution of $\alpha$ in higher density environments (see also [51] for a related discussion of $\Delta G_{N}$ as a function of cosmological environment). In our model, with $\Lambda_{2} \sim \rho_{c}$, cosmological evolution of $\phi$ would not proceed indefinitely into the future but only up to the moment when $\phi$ reaches its low-density minimum at zero. Once again, this behavior is possible only because of the increased coupling strength to matter, $M_{*} \ll M_{\mathrm{Pl}}$.

\section{Nonlinear models}

In this section we re-introduce nonlinear corrections to $V_{\text {eff }}(\phi)$. In fact, several of such models were discussed in the "chameleon" literature [35]-[38], where potentials $V_{\text {eff }}=$ $V(\phi)+\phi \rho$ with different choices of $V(\phi)$ were extensively investigated. As we saw earlier, the quadratic coupling of $\phi$ to matter allows one to escape many stringent constraints, and we intend to keep this feature in this section. To make our discussion more concrete, we limit the form of the potential $V(\phi)$ to have only even powers of $\phi$ and require the coupling to matter to have the same extremum,

$$
V_{e f f}=\frac{1}{4} \Lambda_{4} \phi^{4}+\frac{1}{2}\left(p \Lambda_{2}+q \rho\right) \phi^{2}+\Lambda_{0}
$$

Such a Lagrangian may result, for example, from a discrete symmetry $\phi \rightarrow-\phi$. To ensure overall stability, we must choose $\Lambda_{4}$ to be positive. $p$ and $q$ are taken to be \pm 1 , and we maintain $\Lambda_{2}>0$. Another way to interpret the model (4.25) is to say that we allow for "tachyonic" value of the mass of $\phi$ in (2.8) but ensure an overall stability of the potential by introducing positive $\phi^{4}$ contribution. The cosmological consequences of late time phase transitions were explored in [52].

The most interesting model of this type arises from the choice of parameters that allow 
for the spontaneous breaking of the discrete symmetry, $p=-q$.

$$
p=-1, q=+1 \Rightarrow\left\{\begin{array}{c}
\left\langle\phi^{2}\right\rangle=0 \quad \text { for } \quad \rho>\rho_{c}=\Lambda_{2}, \\
\left\langle\phi^{2}\right\rangle=\Lambda_{4}^{-1}\left(\Lambda_{2}-\rho\right) \text { for } \rho<\rho_{c}=\Lambda_{2} .
\end{array}\right.
$$

It is of course tempting to choose the critical value of matter density $\rho_{c}$ in excess of the average cosmological energy density, so that on average there is a breaking of the discrete symmetry in cosmological environments. However, in matter overdensities of sufficient spatial extent the symmetry is restored, leading to the erasure of the cosmological vacuum expectation value $\langle\phi\rangle$. The difference between masses and couplings between dense and rarefied environments are given by

$$
\frac{\alpha_{r}-\alpha_{d}}{\alpha}=-\xi_{F} \frac{\rho-\Lambda_{2}}{2 \Lambda_{4}} ; \quad \frac{m_{r}-m_{d}}{m}=\frac{\rho-\Lambda_{2}}{2 \Lambda_{4}}
$$

In the broken phase (low-density environments), there is a linear coupling of the Higgs field $\phi-\langle\phi\rangle$ to matter, that creates a contribution to the Newtonian force between two test particles,

$$
\begin{aligned}
U(r) & =G_{N} \frac{m_{N}^{2}}{r}\left(1+\exp \left(-m_{\mathrm{eff}} r\right) \times \frac{2 M_{\mathrm{Pl}}^{2}}{M_{*}^{2}} \frac{\Lambda_{2}-\rho}{\Lambda_{4}}\right) \text { for } \rho<\rho_{c}=\Lambda_{2}, ; \\
m_{\mathrm{eff}}^{2} & =\frac{\Lambda_{2}-\rho}{M_{*}^{2}} .
\end{aligned}
$$

Near the "phase transition", $\rho \simeq \Lambda_{2}$, the range of the force mediated by $\varphi$ becomes infinite. In the unbroken phase, only the quadratic coupling of $\phi$ to matter survives, which as we saw before, significantly relaxes all constraints on the parameters. In other words, the constraint (3.17) is still operative while (3.19) is no longer applicable, provided that $\Lambda_{2}<\rho_{\oplus}$.

As a side remark, we note that there is another interesting spin-off of (4.25) with the following choice of parameters: $p=-q=1$ and $\rho_{\text {star }} \lesssim \Lambda_{2} \lesssim \rho_{\text {core }}$, where $\rho_{\text {core }}$ is a typical density inside a stellar core during a supernovae explosion. In this set-up the scalar field keeps its zero expectation value everywhere except for the extremely dense environments where it is allowed to roll to $\left\langle\phi^{2}\right\rangle \neq 0$. Therefore, for this choice of model parameters, there are no consequences for the terrestrial and cosmological tests of $\Delta \alpha$. There is, however, an interesting possibility that the nucleon mass experiences a shift during the supernova explosion, which in turn may influence the energetics of the explosion and affect the total luminosity (see e.g. [53] where the linear change in strong coupling is discussed in connection with supernova explosions). If the couplings of neutrons and protons to $\phi^{2}$ are different, this model can enhance the environmental dependence of the total SN type Ia luminosity, thus affecting the accuracy with which cosmological parameters can be extracted from supernovae data. The detailed discussion of such possibilities falls outside the scope of our paper. 


\section{Discussion}

On a possibility for new tests of $\alpha(\rho)$ and $m(\rho)$

One possibility to search for the environmental change of masses and couplings caused by a change in density is to try and recreate a low-density environment in the laboratory. The best quality vacuums available today achieve a density at the level of $10^{5}$ particles $/ \mathrm{cm}^{3}$, which creates a matter density comparable to $\mathrm{eV}^{4}$. Taking $\rho_{2} \gg \Lambda_{2} \gg \rho_{1}$, and using the generic solution (3.2), we calculate the resulting shift of $\phi$ and the change in the coupling constant between the center and the walls of a spherical chamber of radius $R$,

$$
\frac{\alpha(r=R)-\alpha(r=0)}{\alpha} \simeq \frac{\xi_{F} \phi_{m}^{2}}{2}\left\{\begin{array}{c}
\frac{1}{36}\left(\frac{R}{\lambda_{v a c}}\right)^{4} \text { for } R / \lambda_{\text {vac }} \ll 1, \\
1 \text { for } R / \lambda_{\text {vac }} \gtrsim 1 .
\end{array}\right.
$$

Similar changes will be experienced by masses of particles. Notice that the parametric dependence of (5.30) is very similar to (3.20), and plugging $\Lambda_{2}$ and $\phi_{m}$ that saturate this constraint we find that

$\frac{\alpha(r=R)-\alpha(r=0)}{\alpha} \sim \xi_{F}\left(\frac{\mathrm{TeV}}{M_{*}}\right)^{2} \times \begin{cases}\left(10^{-17}-10^{-16}\right) \times\left(\frac{R}{1 \mathrm{~m}}\right)^{4} & \text { for } \quad R / \lambda_{\text {vac }} \ll 1, \\ \left(10^{-15}-10^{-14}\right) \times\left(\frac{\lambda_{v a c}}{1 \mathrm{~m}}\right)^{4} & \text { for } \quad R / \lambda_{v a c} \gtrsim 1 .\end{cases}$

These shifts are extremely small, but perhaps are not so far away from the modern capabilities of frequency measurements that can be sensitive to the relative shifts as low as

$10^{-15}$ [54]. Further gain in sensitivity can be achieved with substances that have a large enhancement factors that connect $\Delta \omega / \omega$ with $\Delta \alpha / \alpha[55]$.

To put (5.31) in perspective, we compare this with the result obtained for $\Delta \alpha / \alpha$ between two points separated vertically by $\sim 1 \mathrm{~m}$ in the original Bekenstein model with a massless scalar field. In that model, the coupling of $\phi$ to matter is linear and very weak, and the equivalence principle tests require that $\zeta_{F}^{2}\left(M_{\mathrm{Pl}} / M_{*}\right)^{2}<10^{-6}$ (see e.g. Fig 2 of [8]). In the Bekenstein model, $\Delta \alpha / \alpha=\zeta_{F} \Delta \phi$, where $\Delta \phi$ is the difference in $\phi$ over $1 \mathrm{~m}$ above the surface of the Earth. The solution for $\phi$ is given by

$$
\phi=\frac{\zeta_{m} \rho r^{2}}{6 M_{*}^{2}},
$$

where $\zeta_{m}$ is the coupling of the scalar to matter (this can be obtained easily from the massless limit of the solution given in [39]). In the Bekenstein model, this is absent in the Lagrangian but is induced by the coupling of the scalar to the electromagnetic field in the nucleon. Roughly, one finds $\zeta_{m} \sim 10^{-4} \zeta_{F}$ [8]. Therefore $\Delta \phi$ is simply

$$
\Delta \phi=\frac{\zeta_{m} \rho R_{\oplus} h}{3 M_{*}^{2}}=\frac{10^{-4} \zeta_{F} g h}{3}\left(\frac{M_{\mathrm{Pl}}}{M_{*}}\right)^{2}
$$


where $h=1 \mathrm{~m}$ is the vertical separation of the two points. Putting all factors together one arrives at

$$
\frac{\Delta \alpha}{\alpha}(1 \mathrm{~m}) \sim(g \times 1 \mathrm{~m}) \times 10^{-4} \zeta_{F}^{2}\left(\frac{M_{\mathrm{Pl}}}{M_{*}}\right)^{2} \lesssim 10^{-26} \quad \text { Bekenstein model },
$$

where $g$ is again the free-fall acceleration at the Earth's surface. This is well below any detection sensitivity for the foreseeable future. The difference between (5.31) and (5.34) is an enormous factor of nine orders of magnitude that can be traced back to the fact that equivalence principle is checked at macroscopic distances far better than gravity at distances under $1 \mathrm{~mm}$. Chameleon models do not allow one to make a simple universal estimate of an allowed shift because they depend in a crucial way on the form of the self-interaction potential. Widely discussed chameleon models would not allow for a large spatial variation, even if one departs from the unviersal coupling of chameleon field to matter. This is again related to the extremely tiny values of chameleon field relative to the Planck mass, and $O\left(1 / M_{\mathrm{Pl}}\right)$ size of the chameleon coupling to matter.

Estimates for $\Delta\left(m_{e} / m_{p}\right)$ similar to (5.31) involve a different factor, $\xi_{e}-\zeta_{p}=\xi_{e}-1$. It turns out that this factor can much larger than unity by up to two orders of magnitude. The difference comes about due to relaxed astrophysical constraints on $\xi_{e} / M_{*}^{2}$. Indeed, the rate of $e^{+}+e^{-} \rightarrow \phi \phi$ is additionally suppressed relative to (3.10) by a factor of $\left(m_{e} / T\right)^{2}$, thus allowing for a much larger value of $\xi_{e}$.

If a nonlinear model of the scalar field such as that described by Eq. (4.25) is realized, an artificially created underdensity may lead to the shift of couplings according to (4.27). For the shift to occur, one needs $\rho_{r<R}<\Lambda_{2}<\rho_{r>R}$ and $R>M_{*} \Lambda_{2}^{-1 / 2}$, which would allow for the broken phase to be created within $r<R$ volume.

Astrophysical checks. The possibility of an environmental dependence of coupling constants calls for new tests of $\Delta \alpha(\rho)$ and $\Delta\left(m_{e} / m_{p}\right)(\rho)$. In Bekenstein-type models where couplings evolve in time, there is a clear gain from testing $\alpha$ at maximally available redshifts. On the contrary, in the models discussed here, there is no gain in the size of the effect at large redshift for tests of $\Delta \alpha(\rho)$, and therefore one could search for new test sites within our galaxy. Atomic and molecular absorption lines in the interstellar medium could be an example where extremely narrow lines can be detected. Another beneficial aspects of testing for variations within our galaxy is the possible access to heavier elements where relativistic effects are significantly enhanced. It remains to be seen whether $O\left(10^{-5}\right)$ sensitivity for $\Delta \alpha / \alpha$ achieved in the QSO absorption spectra can be improved upon using galactic lines. 


\section{Conclusions}

We have demonstrated that a scalar coupling to matter can be much stronger than the gravitational coupling in Damour-Polyakov type models. The quadratic nature of coupling to matter allows one to escape the most prohibitive astrophysical and gravitational constraints, as only pair-production or pair-exchange of $\phi$-quanta are allowed. The environmental dependence of masses and coupling constant can come about from the shift in the expectation value of $\phi$ between dense and rarefied environments. We have shown that such shifts could be at a detectable level, and could indeed be probed with astrophysical tests within our galaxy and in laboratory clock comparison experiments.

Acknowledgments. The authors would like to thank Nemanja Kaloper and Nelson Nunes for discussions on chameleon-type models. M.P. would like to acknowledge useful conversations with Dave DeMille, Sara Ellison, Justin Khouri and Adam Ritz. The work of K.A.O. was partially supported by DOE grant DE-FG02-94ER-40823. The work of M.P. was supported in part by NSERC, Canada, and research at the Perimeter Institute is supported in part by the Government of Canada through NSERC and by the Province of Ontario through MEDT.

\section{References}

[1] B. Ratra, P.J.E. Peebles, Phys. Rev. D 37, 3406 (1988); Ap. J. Lett 325, 117 (1988); C. Wetterich, Nucl. Phys. B 302, 668 (1988); R.R. Caldwell, R. Dave and P.J. Steinhardt, Phys. Rev. Lett 80, 1582 (1998) [astro-ph/9708069].

[2] R. S. Van Dyck Jr., P. B. Schwinberg, and H. G. Dehmelt, Phys. Rev. Lett. 59, 26 (1987).

[3] B. Odom, D. Hanneke, B. D’Urso, and G. Gabrielse Phys. Rev. Lett. 97, 030801 (2006); G. Gabrielse, D. Hanneke, T. Kinoshita, M. Nio and B. Odom, Phys. Rev. Lett. 97, 030802 (2006).

[4] J. K. Webb et al., Phys. Rev. Lett. 87, 091301 (2001) [arXiv:astro-ph/0012539]; M. T. Murphy, J. K. Webb and V. V. Flambaum, Mon. Not. Roy. Astron. Soc. 345, 609 (2003) [arXiv:astro-ph/0306483].

[5] C. Wetterich, JCAP 0310, 002 (2003); M. Peloso and E. Poppitz, Phys. Rev. D 68 (2003) 125009 [arXiv:hep-ph/0307379]; D. S. Lee, W. Lee and K. W. Ng, Int. J. Mod. Phys. D 14, 335 (2005) [arXiv:astro-ph/0309316]; W. L. Lee, K. W. Ng and D. S. Lee, Mod. Phys. Lett. A 19, 1089 (2004); N. J. Nunes and J. E. Lidsey, Phys. Rev. D 69, 123511 (2004) [arXiv:astro-ph/0310882]. M. Byrne and C. Kolda, 
arXiv:hep-ph/0402075; P. P. Avelino, C. J. A. Martins and J. C. R. Oliveira, Phys. Rev. D 70, 083506 (2004) [arXiv:astro-ph/0402379]. Y. Fujii and S. Mizuno, Int. J. Mod. Phys. D 14, 677 (2005) [arXiv:astro-ph/0404222]; M. Doran, JCAP 0504, 016 (2005) [arXiv:astro-ph/0411606]; V. Marra and F. Rosati, JCAP 0505, 011 (2005) [arXiv:astro-ph/0501515]; P. P. Avelino, C. J. A. Martins, N. J. Nunes and K. A. Olive, Phys. Rev. D 74 (2006) 083508 [arXiv:astro-ph/0605690]; T. Dent, JCAP 0701, 013 (2007).

[6] M. Livio and M. Stiavelli, Ap. J. Lett. 507 (1998) L13 [arXiv:astro-ph/9808291].

[7] S. J. Landau and H. Vucetich, Astrophys. J. 570, 463 (2002) [arXiv:astro-ph/0005316].

[8] K. A. Olive and M. Pospelov, Phys. Rev. D 65, 085044 (2002) [arXiv:hep-ph/0110377].

[9] H. B. Sandvik, J. D. Barrow and J. Magueijo, Phys. Rev. Lett. 88, 031302 (2002) [arXiv:astro-ph/0107512]; J. D. Barrow, H. B. Sandvik and J. Magueijo, Phys. Rev. D 65, 063504 (2002) [arXiv:astro-ph/0109414]; J. D. Barrow, J. Magueijo and H. B. Sandvik, Phys. Lett. B 541, 201 (2002) [arXiv:astro-ph/0204357].

[10] G. R. Dvali and M. Zaldarriaga, Phys. Rev. Lett. 88, 091303 (2002) [arXiv:hepph/0108217].

[11] T. Chiba and K. Kohri, Prog. Theor. Phys. 107 (2002) 631 [arXiv:hep-ph/0111086].

[12] C. Wetterich, Phys. Lett. B 561, 10 (2003) [hep-ph/0301261]; [hep-ph/0302116].

[13] L. Anchordoqui and H. Goldberg, Phys. Rev. D 68, 083513 (2003) [arXiv:hep$\mathrm{ph} / 0306084]$.

[14] Y. Fujii, Phys. Lett. B 573, 39 (2003) [arXiv:astro-ph/0307263].

[15] E. J. Copeland, N. J. Nunes and M. Pospelov, Phys. Rev. D 69, 023501 (2004) [arXiv:hep-ph/0307299].

[16] S. Lee, K. A. Olive and M. Pospelov, Phys. Rev. D 70, 083503 (2004).

[17] H. Chand, R. Srianand, P. Petitjean and B. Aracil, Astron. Astrophys. 417, 853 (2004) [arXiv:astro-ph/0401094]; R. Srianand, H. Chand, P. Petitjean and B. Aracil, Phys. Rev. Lett. 92, 121302 (2004) [arXiv:astro-ph/0402177].

[18] M. T. Murphy, J. K. Webb and V. V. Flambaum, arXiv:astro-ph/0612407; M. T. Murphy, J. K. Webb and V. V. Flambaum, arXiv:0708.3677 [astro-ph].

[19] E. Reinhold, R. Buning, U. Hollenstein, A. Ivanchik, P. Petitjean and W. Ubachs, Phys. Rev. Lett. 96, 151101 (2006). 
[20] V. V. Flambaum and M. G. Kozlov, arXiv:0704.2301 [astro-ph].

[21] A. I. Shlyakhter, Nature 264 (1976) 340; T. Damour and F. Dyson, Nucl. Phys. B 480, 37 (1996); Y. Fujii et al., Nucl. Phys. B 573, 377 (2000); V. V. Flambaum and E. V. Shuryak, Phys. Rev. D 65, 103503 (2002); C. R. Gould, E. I. Sharapov and S. K. Lamoreaux, Phys. Rev. C 74, 024607 (2006).

[22] K. A. Olive, M. Pospelov, Y. Z. Qian, A. Coc, M. Casse and E. Vangioni-Flam, Phys. Rev. D 66, 045022 (2002) [arXiv:hep-ph/0205269]. K. A. Olive, M. Pospelov, Y. Z. Qian, G. Manhes, E. Vangioni-Flam, A. Coc and M. Casse, Phys. Rev. D 69, 027701 (2004) [arXiv:astro-ph/0309252].

[23] Y. Fujii and A. Iwamoto, Phys. Rev. Lett. 91, 261101 (2003) [arXiv:hep-ph/0309087].

[24] E. W. Kolb, M. J. Perry and T. P. Walker, Phys. Rev. D 33, 869 (1986); R. J. Scherrer and D. N. Spergel, Phys. Rev. D 47, 4774 (1993); L. Bergstrom, S. Iguri and H. Rubinstein, Phys. Rev. D 60, 045005 (1999); K. Ichikawa and M. Kawasaki, Phys. Rev. D 65, 123511 (2002); K. M. Nollett and R. E. Lopez, Phys. Rev. D 66 (2002) 063507; V. F. Dmitriev, V. V. Flambaum and J. K. Webb, Phys. Rev. D 69, 063506 (2004); K. Ichikawa and M. Kawasaki, Phys. Rev. D 69, 123506 (2004); C. M. Muller, G. Schafer and C. Wetterich, Phys. Rev. D 70, 083504 (2004). A. Coc, N. J. Nunes, K. A. Olive, J. P. Uzan and E. Vangioni, Phys. Rev. D 76 (2007) 023511 [arXiv:astroph/0610733]; T. Dent, S. Stern and C. Wetterich, arXiv:0705.0696 [astro-ph].

[25] B. A. Campbell and K. A. Olive, Phys. Lett. B 345, 429 (1995).

[26] R. H. Cyburt, B. D. Fields, K. A. Olive and E. Skillman, Astropart. Phys. 23, 313 (2005).

[27] J. D. Bekenstein, Phys. Rev. D 25 (1982) 1527.

[28] T. Damour, G. W. Gibbons and C. Gundlach, Phys. Rev. Lett. 64, 123 (1990).

[29] For a review see: J. P. Uzan, Rev. Mod. Phys. 75 (2003) 403; V. V. Flambaum, arXiv:0705.3704 [physics.atom-ph].

[30] V.V. Dixit and M. Sher, Phys. Rev. D37 (1988) 1097; P. Langacker, G. Segre and M. J. Strassler, Phys. Lett. B 528, 121 (2002) [arXiv:hep-ph/0112233]; T. Dent and M. Fairbairn, Nucl. Phys. B 653 (2003) 256 [arXiv:hep-ph/0112279]; X. Calmet and H. Fritzsch, Eur. Phys. J. C 24 (2002) 639 [arXiv:hep-ph/0112110]; X. Calmet and H. Fritzsch, Phys. Lett. B 540 (2002) 173 [arXiv:hep-ph/0204258]; T. Damour, F. Piazza and G. Veneziano, Phys. Rev. Lett. 89 (2002) 081601 [arXiv:gr-qc/0204094]; T. Damour, F. Piazza and G. Veneziano, Phys. Rev. D 66 (2002) 046007 [arXiv:hepth/0205111]. 
[31] T. Banks, M. Dine and M. R. Douglas, Phys. Rev. Lett. 88, 131301 (2002)

[32] J. D. Barrow and C. O’Toole, Mon. Not. Roy. Astron. Soc. 322 (2001) 585 [arXiv:astro$\mathrm{ph} / 9904116]$.

[33] T. Damour and K. Nordtvedt, Phys. Rev. Lett. 70, 2217 (1993); T. Damour and K. Nordtvedt, Phys. Rev. D 48, 3436 (1993).

[34] T. Damour and A.M. Polyakov, Nucl. Phys. B 423, 532 (1994) [hep-th/9401069]; Gen. Rel. Grav 26, 1171 (1994) [gr-qc/9411069].

[35] J. Khoury and A. Weltman, Phys. Rev. Lett. 93, 171104 (2004); J. Khoury and A. Weltman, Phys. Rev. D 69, 044026 (2004); S. S. Gubser and J. Khoury, Phys. Rev. D 70, 104001 (2004).

[36] P. Brax, C. van de Bruck, A. C. Davis, J. Khoury and A. Weltman, Phys. Rev. D 70, 123518 (2004); B. Feldman and A. E. Nelson, JHEP 0608, 002 (2006); A. Upadhye, S. S. Gubser and J. Khoury, Phys. Rev. D 74, 104024 (2006).

[37] D. F. Mota and D. J. Shaw, Phys. Rev. Lett. 97, 151102 (2006); D. F. Mota and D. J. Shaw, Phys. Rev. D 75, 063501 (2007).

[38] P. Brax, C. van de Bruck and A. C. Davis, arXiv:hep-ph/0703243; Ph. Brax, C. Vandebruck and A. C. Davis, arXiv:0706.1024 [astro-ph].

[39] J. R. Ellis, S. Kalara, K. A. Olive and C. Wetterich, Phys. Lett. B 228 (1989) 264.

[40] N. Kaloper, arXiv:0706.1977 [astro-ph].

[41] G. G. Raffelt, Ann. Rev. Nucl. Part. Sci. 49, 163 (1999).

[42] N. Arkani-Hamed, S. Dimopoulos and G. R. Dvali, Phys. Rev. D 59, 086004 (1999).

[43] S. Cullen and M. Perelstein, Phys. Rev. Lett. 83, 268 (1999); V. D. Barger, T. Han, C. Kao and R. J. Zhang, Phys. Lett. B 461, 34 (1999).

[44] C. Hanhart, D. R. Phillips, S. Reddy and M. J. Savage, Nucl. Phys. B 595, 335 (2001).

[45] D. J. Kapner, T. S. Cook, E. G. Adelberger, J. H. Gundlach, B. R. Heckel, C. D. Hoyle and H. E. Swanson, Phys. Rev. Lett. 98, 021101 (2007).

[46] E. G. Adelberger, B. R. Heckel, S. Hoedl, C. D. Hoyle, D. J. Kapner and A. Upadhye, Phys. Rev. Lett. 98, 131104 (2007).

[47] R. F. C. Vessot et al, Phys. Rev. Lett. 45, 2081 (1980). 
[48] C. M. Will, Living Rev. Rel. 4, 4 (2001).

[49] T. Ashenfelter, G. J. Mathews and K. A. Olive, Phys. Rev. Lett. 92, 041102 (2004); T. P. Ashenfelter, G. J. Mathews and K. A. Olive, Astrophys. J. 615, 82 (2004); M. G. Kozlov, et al., Phys. Rev. A 70, 062108 (2004).

[50] D. F. Mota and J. D. Barrow, Phys. Lett. B 581, 141 (2004); Mon. Not. Roy. Astron. Soc. 349, 291 (2004). These original claims were subsequently shown to be unfounded in D. J. Shaw and J. D. Barrow, Phys. Rev. D 73 (2006) 123505; D. J. Shaw and J. D. Barrow, Phys. Rev. D 73 (2006) 123506; P. P. Avelino, C. J. A. Martins, J. Menezes and C. Santos, JCAP 0612, 018 (2006).

[51] T. Clifton, D. F. Mota and J. D. Barrow, Mon. Not. Roy. Astron. Soc. 358, 601 (2005).

[52] M. Pietroni, Phys. Rev. D 72, 043535 (2005).

[53] M. Fairbairn, arXiv:astro-ph/9910328.

[54] T. W. Hänsch, Rev. Mod. Phys. 78, 1297 (2006).

[55] V. V. Flambaum, Phys. Rev. Lett. 97, 092502 (2006); V. V. Flambaum and M. G. Kozlov, arXiv:0705.0849 [physics.atom-ph]. 